\title{
Functional dyes from nature: Potentials for technical applications
}

\author{
N. Hampp and A. Silber \\ University of Marburg, Dept. for Physical Chemistry, D-35032 Marburg, Germany
}

\begin{abstract}
Biological photochromes have been optimized in their functional properties during evolution. To use them like 'blue prints' for the construction of new materials is attractive. Genetic engineering is a powerful tool for the controlled modification of the physical properties of biopolymers according to the demands of technical applications. We studied this approach with the biological photochrome bacteriorhodopsin (BR) and demonstrated that mutated BRs may successfully be used in optical media for information storage as well as for optical phase-conjugation, holographic interferometry and pattern recognition.
\end{abstract}

The interaction between light and matter is fundamental for the living nature. Plants, bacteria and algae use sunlight for the fixation of $10^{11}-10^{12}$ tons of carbon dioxide per year during photosynthesis. Besides assimilation vision is the other important area where light sensitive molecules play key roles. For technical applications in the fields of solar energy conversion, optical sensing and (electro-)optical information processing biological photochromic materials are worth a closer look. Table 1 gives a survey of some important biological photochromes and their natural function. In contrast to man-made photochromic materials, which may be syntheticaly optimized considering one or two functional parameters, these natural photochromes represent the 'best fit' to a large set of parameters. Trying to use these highly developed materials for technical applications seems to be a straightforeward approach. However, they either are very complex or they function only in cooperation with several other biopolymers. Only the more simple 'oldfashioned' models from archaebacteria, like bacteriorhodopsin, can be really investigated for their technical usability today.

TABLE 1. Biological photochromes

\begin{tabular}{lll}
\hline Photochrome & Chromophore & Natural function \\
\hline photoreactive center & chlorophyll & conversion of light energy \\
phytochrome & phytochromobilin & photomorphogenesis \\
rhodopsin & 11 -cis-retinal & perception of light \\
photoactive yellow protein (PYP) & p-coumaric acid & phototaxis \\
bacteriorhodopsin (BR) & all-trans retinal & light-driven proton pump \\
halorhodopsin (HR) & all-trans retinal & light-driven chloride pump \\
sensorhodopsin (SR) & all-trans retinal & phototaxis \\
\hline
\end{tabular}

Bacteriorhodopsin occurs in extremely halophilic bacteria in the form of a two-dimensional crystal. In this form it shows an exceptional stability which makes it a preferred candidate for such an investigation. 


\section{STRUCTURE AND FUNCTION OF BACTERIORHODOPSIN}

Bacteriorhodopsin (BR) is found in two-dimensional crystalline patches inside the cell membrane of Halobacterium salinarium (former Halobacterium halobium) which are named purple membranes due to their intense color (1). BR belongs to the family of retinal proteins. A retinylidene residue is covalently attached to the protein moiety via lysine-216. BR is a light-driven proton pump and forms together with a membrane-bound ATP-ase the halobacterial photosynthetic system. By the action of BR

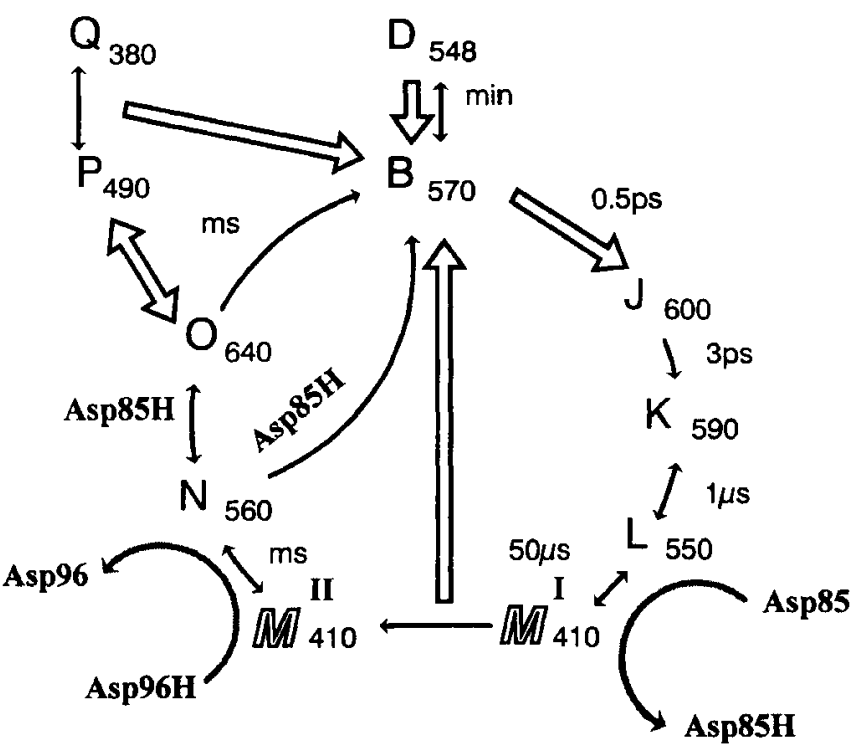

Fig. 1 Photochemical and thermal conversions of bacteriorhodopsin. light energy is converted into the chemical energy of a proton gradient which is further utilized for ATP generation. After photochemical excitation of a BR molecule a cyclic sequence of intermediates is observed $(2,3)$. In Fig. 1 the intermediates of the BR photocycle are given by the common single letter code with the absorption maxima as subscripts. Outlined arrows represent photochemical steps, thin solid arrows indicate thermal conversions. Thick grey arrows are related to the reversible de-/reprotonation of the Schiff base linkage via aspartic acids (Asp) 85 and 96. Protonation of their carboxylic group is indicated by the letter ' $\mathrm{H}$ ' directly following, e.g. Asp85H. Only in the M-state the Schiff base linkage is deprotonated. The light-driven proton transport from the cytoplasma to the extracellular medium is intimately coupled to the photochromism of the BR molecule, i.e. its reversible color change. The photochromism of the BR molecule is a 'symptom' of the molecular changes occuring during the vectorial proton transport but itself it has no biological relevance for the halobacterial cell. However, for possible technical applications of BR, the light-controlled change of the absorption state is the key feature. The photochromic properties of BR may be used in optical information processing techniques. Its excellent thermal, chemical and photochemical stability as well as the fast $(0.5 \mathrm{ps})$ and efficient $(\Phi \geq 0.64)$ initial photochemical reaction (4) of this biological photochrome are attractive properties for the construction of light-sensitive media for optical information processing. However, the various optical techniques require optical media with specifically adapted and widely differing optical properties. This wide range cannot be covered by the $\mathrm{BR}$ type alone which is found in nature, so-called wildtype $\mathrm{BR}$.

Genetic engineering of the protein part BR, i.e. bacterio-opsin, and biotechnological production of substantial amounts of mutated BRs are the keys for the utilization of this attractive water soluble and highly reversible photochromic compound (5). The properties of BR which are desired to be preserved and those which are intended to be modified are listed in table 2 .

TABLE 2. Properties of BR which must be left unchanged and those which should be or may be changed during the modification and optimization of the protein.

The protein modifications

must leave unchanged the systems

may / should affect the systems

- photochromism

- excellent reversibility

- efficient photochemistry
- proton pumping properties

- thermal relaxation rates

- spectral properties of the intermediates 
BR is one of the best investigated membrane proteins. The structure-function relation available is detailed enough to serve as the starting point for the attempt to vary the photophysical properties of BR by modifying the interaction of the retinylidene residue and the amino acid cage formed by the bacterio-opsin (6). Minor changes in the amino acid sequence are desired, which leave the positive properties of BR unchanged (see Table 2) and at the same time allow to modify the photochemical properties of the molecule. By this method mutated BRs for long-term data storage at room temperature (7) as well as transient optical recording, e.g. for optical phase-conjugation (8), real-time pattern recognition (9) and real-time interferometry (10) have been generated. In Fig. 2 the modifications obtained in the photocycle are sketched (11) and the absorption maxima of the most important intermediates are shown.

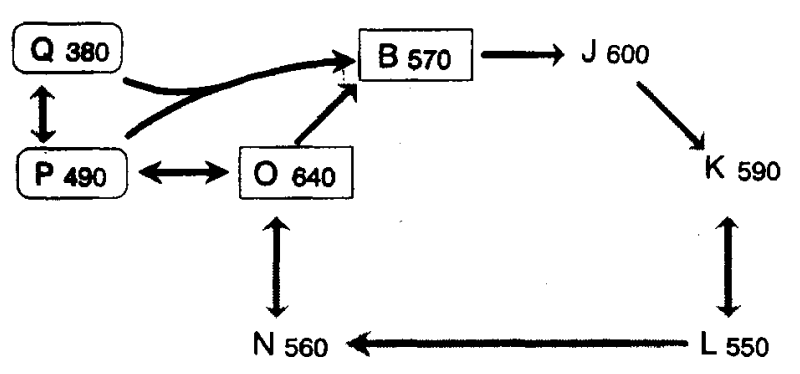

Fig. 2a Modified BR for long-term data storage. By replacement of Asp85 by Asn a shortcut in the photocycle is introduced which leads to an enhanced population of an O-like state. From there the photochemical formation of 9-cis retinal is obtained with red light.

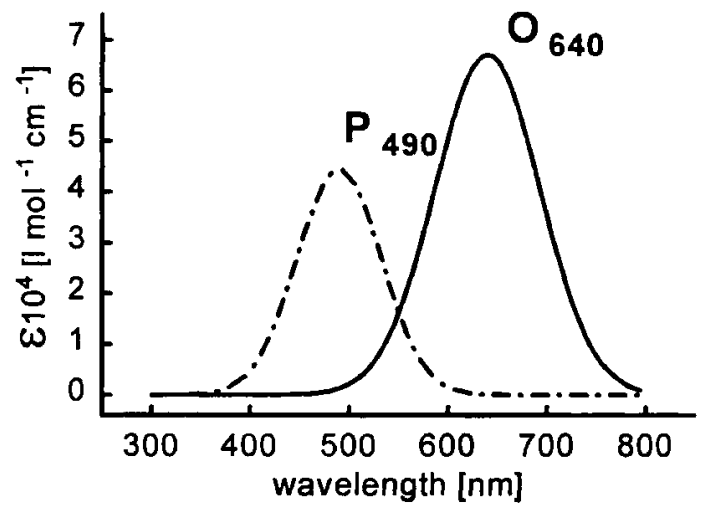

Fig. 2b Absorption spectra of the initial (solid line) and 'bleached' state (broken line). The $\mathrm{P}_{490}$ state contains 9 -cis retinal. This configuration is at room temperature thermally stable in BR but may be photochemically erased with blue-green light.

In principle, such BR materials are suitable for long-term photochromic information storage. These materials demonstrate the usefulness of genetic engineering in material science but from the viewpoint of application they are less important.

In applications where reversibility is required mutated BRs may be the best photochromics available today. The photochromic properties of BR for real-time applications are enhanced by the removal of Asp96. Thereby the lifetime of the M-state is drastically increased and the late intermediates (see Fig. 3a) only reach neglegible populations. For example, in real-time pattern recognition, where two independent TV-frame rate (video) signals are processed, such BR-films are currently unchallenged by classical media. The number of holograms written, read-out and erased in an 12 hrs working session exceeds one million holograms. A number which is approximately one order of magnitude too high for synthetic photochromic materials. The

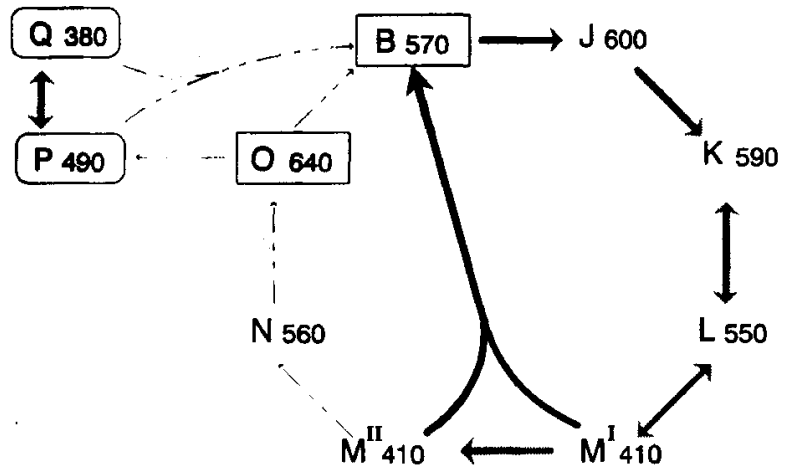

Fig. 3a Modified BR for transient optical recording. An increasing lifetime of the Mstate causes that late intermediates and side branches are only very little populated.

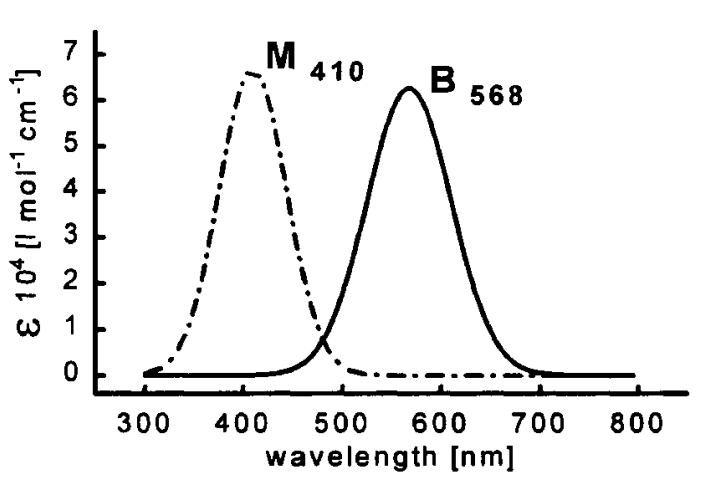

Fig. 3b Absorption spectra of the initial state (solid) and the longest living intermediate (broken line). Recording and erasing on a millisecond timescale is done with blue and yellow light. 
TABLE 3. Holographic properties of BR films

\begin{tabular}{llll}
\hline spectral range & $410-680 \mathrm{~nm}$ & light sensitivity & $5-30 \mathrm{~mJ} / \mathrm{cm}^{2}$ \\
resolution & $z 5000$ lines $/ \mathrm{mm}$ & polarization recording & $\begin{array}{l}\text { possible } \\
\text { optical density }\end{array}$ \\
bleaching ratio & $1-20\left[\mathrm{OD}_{570}\right]$ & reversibility & $\geq 10^{6} \mathrm{cycles}$ \\
index of refraction & 1,47 & film thickness & $10-500 \mu \mathrm{m}^{2}$ \\
refraction index change & $10^{-3}-10^{-2}$ & rise and decay times & $\mathrm{msec}-\mathrm{sec}$ \\
diffraction efficiency & $1-7 \%($ typ. $2-3 \%)$ & aperture & unlimited \\
\hline
\end{tabular}

\footnotetext{
${ }^{1}$ chromophore anisotropy $20: 1$

${ }^{2}$ typically $20 \mu \mathrm{m}$

${ }^{3}$ commercially available $50 \times 50 \mathrm{~mm}^{2}$
}

excellent reversibility of BR allows to perform this task without any detectable change of its photochromic properties. In Table 3 the optical and holographic properties of BR-films for transient recording are summarized.

\section{APPLICATION OF BR-FILMS IN INTERFEROMETRY}

Holographic pattern recognition is an application where all the positive features of BR-films are used. Since no amplification through any step of chemical or thermal development occurs in the BR-films, the light sensitivity in the $\mathrm{mJ} / \mathrm{cm}^{2}$-range is limiting in some applications. In particular holographic interferometry of reflective objects is such an application. We investigated which practical limitations occur. In Fig. 4 a typical homodyne setup is shown. The beam from a $200 \mathrm{~mW}$ frequency-doubled NdYAG laser illuminates the object through a beam expander (BE). A small fraction of the laser beam is decoupled and serves as a reference beam to form reflection type holograms with the light scattered by the object and collected by the photo objective (PO). The object beam is turned off for a short time interval and the synchronized CCD camera records the reflection type interferogram during this period.

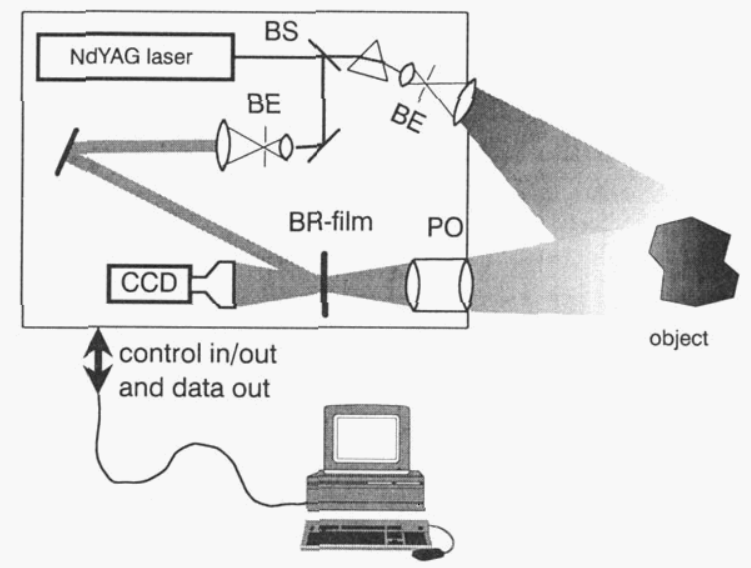

Fig. 4 Interferometric setup with BR-films.

In Fig. 5 several examples of interferograms taken from small mechanical parts are presented. The interferograms shown represent the output of the system's CCD camera. No electronic post processing was applied. For the calculation of the absolute deformation phase-shift interferograms are a commonly used technique (see Fig. $5 \mathrm{~g}-\mathrm{h}$ ). Interferometry of reflective objects by means of BR-films as a recording medium is limited severely by the available light energy. With a $200 \mathrm{~mW}$ source like shown in Fig. 4 objects with dimensions up to about $10 \mathrm{~cm}$ may be analyzed in real-time. 


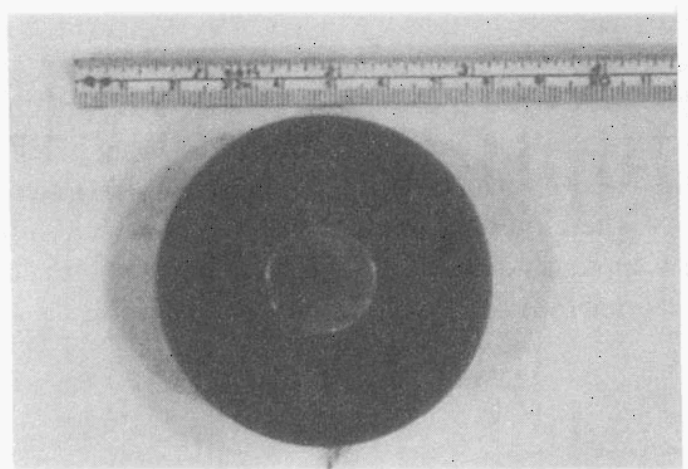

C

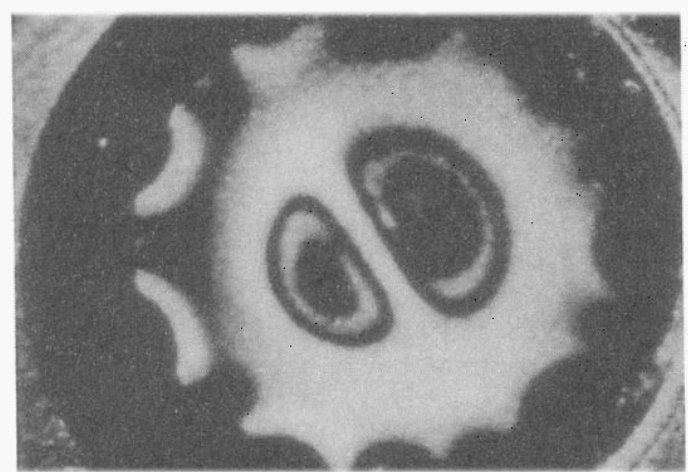

e

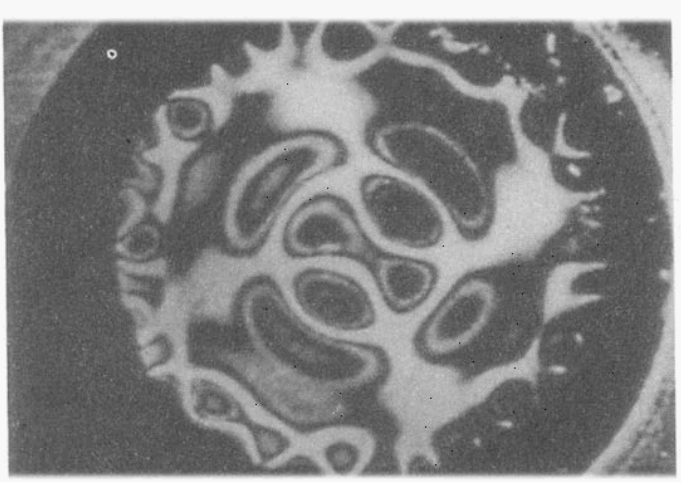

g

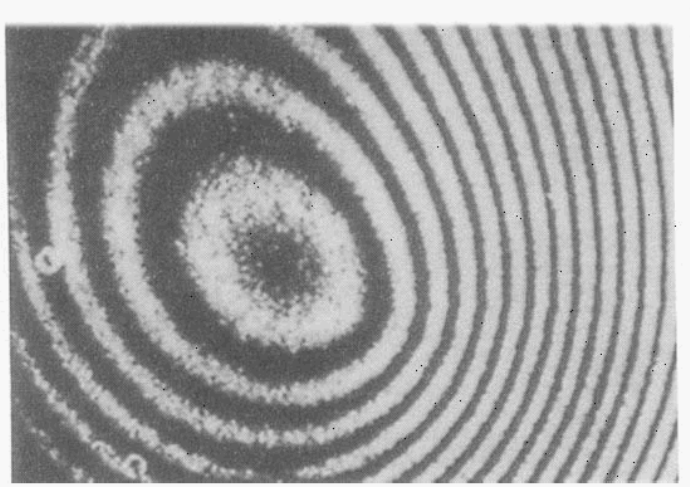

b

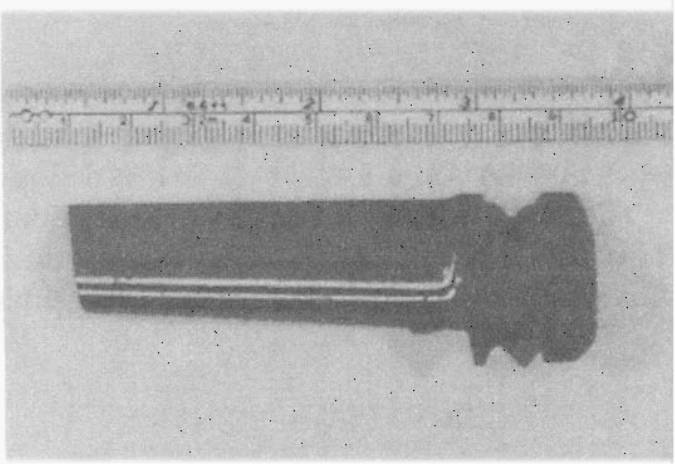

$\mathrm{d}$

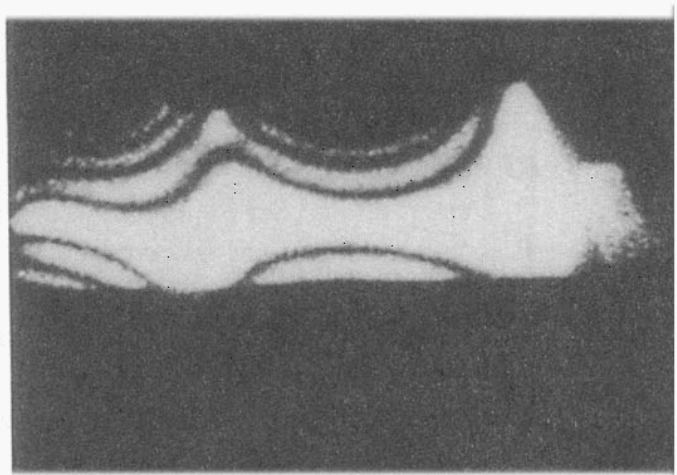

f

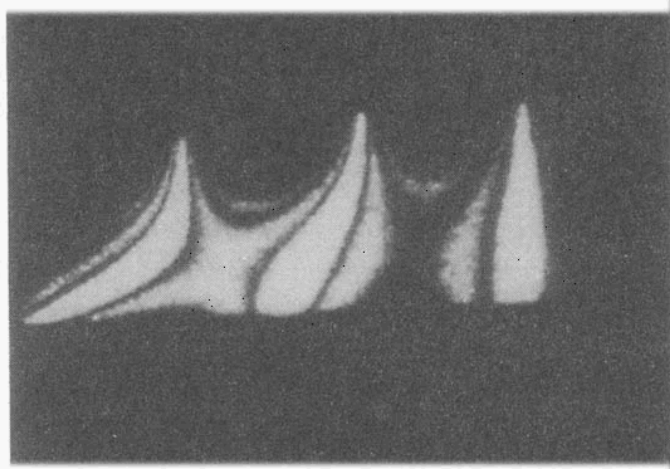

h

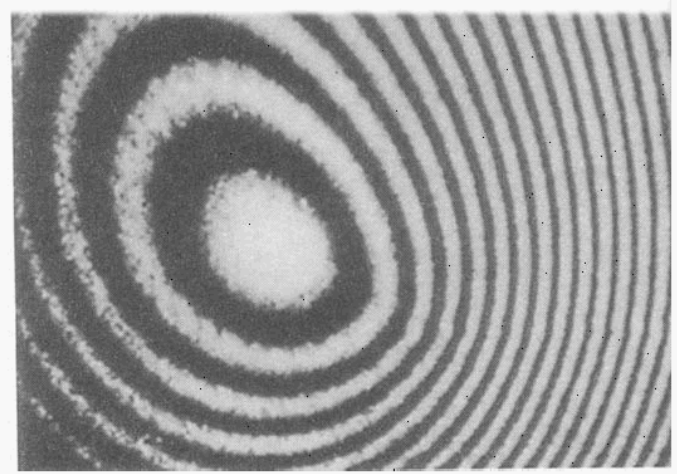

Fig. 5 Interferometry with BR-films. ( $a, b)$ Photographs of investigated objects, $i$. e. a piezo buzzer and a turbine blade. ( $c$ - $f$ ) Time-average interferograms of the objects shown above, recorded at different excitation frequencies. $(\mathrm{g}, \mathrm{h})$ Phase-shift interferograms of the same metal plate recorded simultaneously in the same BR-film. 


\section{SUMMARY}

It has been demonstrated by several groups that wildtype BR as well as chemically or genetically modified $B R$ are useful media for different optical information processing techniques (12 - 15). Engineering of BR by genetic methods is a useful tool for the design of BR-based optical media with a realistic potential for technical applications. The reported results from BR, i.e. the modification of this evolutionary optimized photochromic protein by genetic engineering, mark a new approach in material science which may also serve as a model for the technical utilization of other biological macromolecules.

\section{ACKNOWLEDGEMENTS}

This work was done under BMBF-contract Nr. 0319231. Stimulating discussions with D. Oesterhelt and C. Bräuchle and the experimental assistance of R. Schmid are gratefully acknowledged.

\footnotetext{
REFERENCES

1. D. Oesterhelt and W. Stoeckenius. Nature (London), New Biol. 233, 149 (1971).

2. T. Kouyama et al. Adv. Biophys. 24, 123 (1988).

3. G. Varo and J. K. Lanyi. Biochem. 30, 5008 (1991).

4. R.A. Mathies et al. Annu. Rev. Biophys. Biophys.Chem. 20, 491 (1991).

5. B. F. Ni et al. Gene 90, 169 (1990).

6. R. Henderson et al. J.Mol.Biol. 213, 899 (1990).

7. N. Hampp et al. J.Phys.Chem. 96, 4679 (1992).

8. D. Zeisel and N. Hampp. Opt.Lett. 19, 1412 (1994).

9. R. Thoma and N. Hampp. Opt.Lett. 19, 1364 (1994).

10. N. Hampp et al. Mat.Res.Soc.Symp.Proc. 330, 269 (1994).

11. A. Popp et al. Biophys.J. 65, 1449 (1993).

12. R.R. Birge, Sci.Am. 272(3), 66 (1995).

13. D. Oesterhelt et al. Quart.Rev.Biophys. 24, 425 (1991).

14. N. Hampp et al. MRS Bulletin, 17, 56 (1992).

15. T. Miyasaha et al. Science 255, 342 (1992).
} 